\title{
Selbstsozialisation und Medienbildung
}

\author{
Tilmann Sutter
}

\section{Einleitung: Medienbildung - Probleme und Desiderate}

Eine Theorie der Medienbildung mit ihren besonderen Fragestellungen steht im Kontext vielfältiger Theorien der Medienkompetenz und der Mediensozialisation. Man wird also bei Fragen der Medienbildung um eine Berücksichtigung dieser Forschungsfelder kaum herumkommen, wobei einige zentrale Probleme und Desiderate in den Blick kommen. Grundsätzlich ist nach dem Verhältnis einer Theorie der Medienbildung zu Modellen der Medienkompetenz zu fragen. Hier wird der Begriff der Medienbildung zunehmend den vielfach behaupteten Medienkompetenzen gegenübergestellt, insofern Medienkompetenzen im Sinne von Qualifikationen, Bildungsstandards, Wissensbeständen oder anderen strategisch einsetzbaren bzw. gesellschaftlich abverlangten Fertigkeiten konzipiert werden, die ebenso Fragen der normativen Festlegung wie Probleme der empirischen Messbarkeit stellen (vgl. Herzig/Grafe 2010; Jörissen 2011). Dieser Stand der Diskussion kommt nicht von ungefähr, denn ein erstes allgemeines Problem von Lern-, Sozialisations- und Kompetenztheorien stellen Fokussierungen auf Entwicklungsresultate dar, die wesentlich einfacher zu untersuchen sind als die entsprechenden Fragen des Erwerbs dieser Resultate. Gerade im Bereich der Medien können viele Kompetenzen und Lernresultate behauptet werden, die sowohl Voraussetzung als auch Ergebnis des Umgangs mit Medien sind, deren Plausibilität und Relevanz in der Rekonstruktion von Erwerbsprozessen allerdings erst noch zu zeigen wären. Es geht darum, ebenso den allgemeinen Begriff der Bildung wie auch den spezifischen Begriff der Medienbildung von der Ausrichtung auf Bildungsstandards und auf Lernresultate abzulösen: Auf dieser Linie zielen die folgenden Ausführungen darauf ab, einige Bausteine einer Theorie der Medienbildung zu umreißen. Dabei gehen die Erörterungen davon aus, dass zentrale Probleme und Desiderate einer Theorie der Medienbildung auf die Frage nach Prozessen der Medienbildung bezogen werden können (vgl. Marotzki/Jörissen 2010). Die Gründe hierfür werden deutlich, wenn man Probleme von Theorien und Untersuchungen 
von Medienkompetenzen betrachtet, die sich auf Analysen feststellbarer Lernresultate im Umgang mit Medien konzentrieren. Eine Kritik dieser kompetenztheoretischen Perspektive, die in das Desiderat einer Theorie des Erwerbs von Medienkompetenzen mündet, bildet einen ersten Baustein einer Theorie der Medienbildung (2).

Eine Theorie des Erwerbs von Medienkompetenzen fragt nicht nur, was im Umgang mit Medien gelernt und beherrscht wird, sondern auch, wie und in welchen Prozessen sich dies vollzieht. Grundlage einer Theorie des Erwerbs von Medienkompetenzen liefert mithin eine Theorie der Mediensozialisation, die als ein zentraler zweiter Baustein einer Theorie der Medienbildung zu erörtern ist. Dabei kommt das Verhältnis von Selbst- und Fremdsozialisation in den Blick, mit dem sowohl allgemeine Sozialisationstheorien als auch Mediensozialisationstheorien unterschieden werden können. In verschiedener Weise werden Aspekte der Selbstund Fremdsozialisation zueinander in Beziehung gesetzt, womit eine zentrale Grundlage des Erwerbs von Medienkompetenzen geklärt werden kann. Neben vielfältigen Beobachtungen einer zunehmenden Pluralisierung und Flexibilisierung von Lebens- und Identitätsentwürfen in der modernen Gesellschaft, die mehr und mehr in Selbstregie entwickelt werden, stellt eine konstruktivistische Theorie der (Medien-)Sozialisation aus einer grundlegenden theoretischen Perspektive den Aspekt der Selbstsozialisation heraus. Damit wird aus soziologischer Sicht der Zusammenhang von Selbstsozialisation und funktionaler Ausdifferenzierung der Gesellschaft erkennbar (3). Vor dem Hintergrund der wachsenden Bedeutung neuer computergestützter Medien wird diese Tendenz auch im Rahmen des Wandels von Massenmedien zu neuen Medien verstärkt. Damit wird eine zentrale These für eine Theorie der Medienbildung gewonnen: Der Umgang mit neuen Medien stellt mehr als bisher auf Prozesse der Selbstsozialisation ab (4). Auf der genannten Linie kann die Soziologie Beiträge $\mathrm{zu}$ einer Theorie der Medienbildung liefern, die als eine Art Reflexionstheorie fungiert. Diese Reflexionstheorie prüft unter dem Bezugsproblem der Bedeutung von Selbstsozialisation in der modernen, funktional ausdifferenzierten Gesellschaft sowie des Medienwandels die Möglichkeiten und Leistungen von Medien und des Umgangs mit Medien für subjektive Bildungsprozesse (5).

\section{Kritik kompetenztheoretischer Perspektiven}

Während der allgemeine Begriff der „Kompetenz“ auf vielfältigste Weise eine Fülle von Diskursen in Wissenschaft, Erziehung, Politik usw. 
durchzieht, ist der speziellere Begriff der Medienkompetenz zum Schlagwort für das Lernen in der Informationsgesellschaft geworden. Aktuell machen vor allem die Möglichkeiten, Risiken und Gefahren des Internet den Stellenwert eines kompetenten Umgangs mit Medien deutlich. Medien bestimmen zunehmend unseren Zugang zur Welt und eröffnen neue Wege der Information, Kommunikation und Unterhaltung. Wenn man den Begriff der Medienkompetenz im Zusammenhang von Bildung und Erziehung betrachtet, kann die Abhängigkeit der Gesellschaftsmitglieder von Medien und deren Rolle in der Gesellschaft durchaus unterschiedlich eingeschätzt werden. Berücksichtigt man eher die Einflüsse und die Eigenlogik der Medien, wird der Raum für aktive Deutungs- und Aneignungsprozesse der Rezipienten im Umgang mit Medien systematisch eingeschränkt. Dagegen kommen aus einer rezeptionsorientierten Perspektive sowohl die Freiräume als auch die inneren und äußeren Voraussetzungen und Bedingungen subjektiver Umgangsweisen mit Medienangeboten in den Blick (vgl. Charlton 1997). Prozesse der Bildung und Erziehung schließen einen aktiven Umgang mit Medien ein, der erst gelernt werden muss und in dem bestimmte Fähigkeiten erworben werden. Um welche Fähigkeiten es sich dabei handelt, dies ist die Frage, um die Untersuchungen der Medienkompetenz vornehmlich kreisen. Wenn auch die überragende Bedeutung von Medienkompetenzen kaum in Zweifel $\mathrm{zu}$ ziehen ist, stellen sich einer Theorie der Medienkompetenz vor allem zwei Probleme: Die Verbreitung des (Medien-)Kompetenzbegriffs führt erstens dazu, dass der Begriff unscharf und mehrdeutig wird. Und die Frage, was im Umgang mit Medien gelernt und beherrscht werden muss, drängt zweitens die Frage in den Hintergrund, wie dies geschieht. Theorien der (Medien-)Kompetenz sind, mit anderen Worten, zu sehr auf statische Lernresultate fokussiert. Diese beiden Probleme seien nachfolgend kurz umrissen (ausführlicher hierzu Sutter/Charlton 2002).

Die Unschärfe des Kompetenzbegriffs macht es notwendig, ihn für die eigenen Zwecke, z.B. eine Theorie der Medienbildung zu präzisieren. Hierbei ist es hilfreich, sich die Geschichte des Begriffs „Kompetenz“ zu vergegenwärtigen. Im vorliegenden Zusammenhang sind vor allem zwei Phasen der Entfaltung von Kompetenztheorien relevant: die sprachwissenschaftliche Bestimmung des Kompetenzbegriffs während der 70er Jahre und seine Übernahme in unterschiedliche Entwicklungs- und Sozialisationstheorien. In der Sprachwissenschaft ist der Begriff der Kompetenz in Abgrenzung zu Performanz aus der Linguistik Noam Chomskys geläufig. Sprachkompetenz im Sinne Chomskys meint das intuitive Regelwissen, über das die Subjekte verfügen. Dabei wird von einem idealen Sprecher-Hörer mit optimaler Sprachkenntnis ausgegangen, deren 
Anwendung keinen einschränkenden Faktoren unterliegt. Diese Faktoren bestimmen die Performanz, also die Sprachverwendung (vgl. Chomsky 1972). Der Begriff der Sprachkompetenz zielt auf angeborene, individuelle Prädispositionen, die auf einer synchronen Ebene liegen. Dagegen steht in sozialisationstheoretisch gewendeten Kompetenztheorien die Frage des konstruktiven Erwerbs subjektiver und sozialer Kompetenzen im Mittelpunkt. Es handelt sich um Theorien allgemeiner kognitiver, sozialer und moralischer Kompetenzen. Zwar wandelt sich Kompetenz damit von einem synchronen zu einem diachronen Konstrukt. Tatsächlich erfassen Kompetenztheorien allerdings nicht so sehr Entwicklungsprozesse, sondern sie fertigen strukturanalytische Beschreibungen von Stufenmodellen der kognitiven, sozialen und moralischen Entwicklung (also von Entwicklungsresultaten) an. Mit der Aufnahme des Kompetenzbegriffs in die soziologische Sozialisationsforschung wird ein neuer Fokus gesetzt: Subjekte entwickeln ihre Kompetenzen in sozialen Interaktionen und im Rahmen sozialer Lernprozesse. Jürgen Habermas (1995) spricht in diesem Zusammenhang von Rollenkompetenz, Interaktionskompetenz oder aber auch von kommunikativer Kompetenz. Der subjektive Kompetenzerwerb setzt aus dieser Sicht die Teilnahme an Kommunikationsprozessen voraus. Der Begriff der kommunikativen Kompetenz hat sich weit vom ursprünglichen Begriff der Kompetenz entfernt und reagiert auf eine zentrale Kritik am Kompetenzbegriff, der zufolge die aktiven Erwerbsstrategien, vor allem aber auch die sozialen Entwicklungsbedingungen der Subjektbildung kompetenztheoretisch nicht expliziert werden können. Darüber hinaus waren die Kompetenztheorien grundlegenden Kritiken ausgesetzt, die sich u.a. gegen eine entwicklungs- und sozialisationstheoretische Übernahme des Begriffs der Kompetenz richteten. Diesen Kritiken zufolge ist der Begriff der Kompetenz nativistisch, synchron und individuell angelegt und eignet sich deshalb nicht für diese Übernahme. So können weder die subjektiven Erwerbsstrategien noch die sozialen Bedingungen der Subjektbildung kompetenztheoretisch untersucht werden. Letztlich war man überwiegend mit Beschreibungen vermuteter Kompetenzen und weniger mit Prozessen des Kompetenzerwerbs befasst (vgl. Sutter 2009: 186ff).

Auch Medienkompetenzen werden als Lernresultate beschrieben und weisen eine große Vielfalt auf (zum Überblick vgl. Herzig/Grafe 2010): Medienkompetenzen umfassen Fähigkeiten des Verständnisses medialer Angebote. Dieses Verständnis erfordert u.a. sprachliches, formallogisches und sozialkognitives Regelwissen zur Rekonstruktion der medialen Sinnstrukturen. Medienkompetenzen bestehen darüber hinaus in der praktischen Beherrschung von Medien. Medienkompetenzen ermöglichen den 
Einsatz von Medien zur Lösung von schulischen oder beruflichen Aufgaben ebenso wie zur Gestaltung von Freizeit. Medienkompetenzen zeigen sich darüber hinaus in Fertigkeiten der Herstellung von Medienprodukten. Medienkompetenzen liegen schließlich der Bewertung und kritischen Beurteilung von Medien zugrunde. Medienkompetenzen werden so nicht nur auf allgemeine subjektive Fähigkeiten, sondern auch auf bestimmte Lebensbereiche und Aufgaben bezogen. Darüber hinaus kommen weitere Dimensionen von Medienkompetenz (etwa Anschlusskommunikationen bzw. kommunikative Verarbeitungen von Medienerfahrungen) in den Blick. Auch Bezüge auf verschiedene Medienarten sind zu berücksichtigen.

Aus kompetenztheoretischer Sicht kann man nicht nur (mehr oder weniger plausible) Medienkompetenzen, sondern auch einen entwicklungs- und altersabhängigen Umgang von Subjekten mit Medienangeboten beschreiben (vgl. Theunert 1999). Subjektive Kompetenzen bedingen altersspezifisch gegebene Möglichkeiten, Medienangebote zu verstehen und sie für die Persönlichkeitsentwicklung zu nutzen. Damit ist auch eine Grundlage gegeben, eine altersabhängige Angemessenheit medialer Angebote zu rekonstruieren. So verfügen Subjekte über bestimmte sozialkognitive Mittel, um Medienfiguren und Mediengeschichten zu verstehen (vgl. Sutter 1999). Aus kompetenztheoretischer Sicht gibt es einen im Entwicklungsverlauf sich ändernden Umgang mit Medienangeboten. Empirisch ist es die Praxis dieses Umgangs (und nicht die postulierten Kompetenzen), die sich beobachten und rekonstruieren lässt. Dieses Verhältnis von postulierter Kompetenz und Performanz (als vielfach bedingter, praktischer Umsetzung der Kompetenz) bildet seit jeher ein zentrales Problem der Erforschung von Kompetenzen. Viel zu selten ist daraus der Schluss gezogen und auch konsequent umgesetzt worden, subjektive Voraussetzungen und soziale Bedingungen der Mediennutzung darauf hin zu untersuchen.

Damit rückt ein zentrales Desiderat einer Theorie der Medienkompetenz in den Mittelpunkt: Sie sollte sich nicht darauf beschränken, mit der Behauptung dieser oder jener Medienkompetenzen nur die Resultate individueller Lernprozesse im Blick zu haben. Ein bloßer Lernzielkatalog, so plausibel er auch erscheint, macht nicht deutlich, vor welche Herausforderungen der Umgang mit Medien die Nachwachsenden stellt und wie diese Aufgaben bewältigt werden. Es gilt mithin, sowohl soziale Bedingungen als auch die prozessuale Dynamik von Entwicklungs- und Sozialisationsprozessen im Feld der Medienkompetenzen zu rekonstruieren. Denn genau dies kann man in vielen Bereichen der Erforschung subjektiver und sozialer Kompetenzen beobachten: Es können viele (Me- 
dien-) Kompetenzen behauptet werden, deren Gültigkeit sich jedoch erst mit der Rekonstruktion ihrer Bildungsprozesse zeigt. Eine Theorie der Medienbildung greift notwendigerweise über eine Theorie der Medienkompetenz hinaus, indem sie Prozesse der Medienrezeption und der Mediensozialisation einbezieht (vgl. Pietraß 2009). Sie wird deshalb nicht umstandslos auf bestehende Modelle der Medienkompetenzen zugreifen können. Wie Kompetenzen erworben werden, wird schon seit langer Zeit debattiert, wobei diese Diskussion in den letzten Jahren im Bereich der Mediensozialisation als Verhältnis von Fremd- und Selbstsozialisation fortgeführt wird.

\section{3. (Medien-)Selbstsozialisation und gesellschaftliche Differenzierung}

Das Verhältnis von Fremd- und Selbstsozialisation kann als Orientierungsrahmen dienen, innerhalb dessen unterschiedliche Auffassungen eingeschätzt werden, wie Medienkompetenzen erworben werden. In sozialwissenschaftlichen Medienforschungen ist dieser Orientierungsrahmen wiederum eingespannt in eine traditionsreiche Auseinandersetzung zwischen Medienwirkungsforschungen, die von einem starken Einfluss der Medien, und Mediennutzungsforschungen, die von aktiven Gestaltungsmöglichkeiten bzw. Selbststeuerungspotentialen der Medienrezipienten ausgehen. Im ersten Fall werden eher Aspekte der Fremdsozialisation im Umgang mit Medien betont, im zweiten Fall wird man eher die Rolle der Selbstsozialisation betonen. Sicherlich heben medienpädagogische Perspektiven, insbesondere im Bereich der Massenmedien, eher die Bedeutung von Fremdsozialisation hervor, etwa wenn Möglichkeiten der gezielten Förderung von Fähigkeiten der Mediennutzung, aber auch Einflüsse z.B. des familiären Umfeldes diskutiert werden, denen die nachwachsenden Mediennutzer ausgesetzt sind (vgl. Süss 2004: 70f.). Allerdings rückt die Bedeutung von Prozessen der Selbstsozialisation im Umgang mit Medien schon seit längerem in den Blick, sei es als notwendige Ergänzung von Prozessen der Fremdsozialisation, sei es als grundlegende alternative Perspektive im Bereich der Mediensozialisationstheorien. Hier könnte der aktuelle Medienwandel von älteren Massenmedien zu neueren ,,interaktiven" Medien der Internetkommunikation als Taktgeber einer Akzentverschiebung fungieren (vgl. Sutter 2010: 181ff.). Wenn und insoweit Kinder und Jugendliche den Umgang mit neuen Medien zunehmend in Eigenregie erlernen, weil Elternhaus und Schule hier nicht mehr als kompetente Begleiter zur Verfügung stehen, werden Prozesse der Selbstsozialisation 
verstärkt zu beachten sein. Diese gesteigerte Bedeutung von Selbstsozialisation wird auch für eine Theorie der Medienbildung folgenreich sein, insofern sich hier größere Erfordernisse eines reflexiven Umgangs mit Medien mit einem entsprechenden Orientierungsbedarf abzeichnen.

Treten wir aber einstweilen nochmals einen Schritt zurück und fragen allgemein nach dem Verhältnis von Theorien der Selbst- und Fremdsozialisation. Ein allgemeines Bezugsproblem, an dem eine Systematisierung von Sozialisationstheorien ausgerichtet werden kann, stellt die Gewichtung unterschiedlicher Aspekte der Fremd- und Selbstsozialisation dar: Je mehr direkte äußere Einfluss-, Instruktions- und Eingriffsmöglichkeiten auf die Subjekte angenommen werden, desto mehr werden Prozesse der Fremdsozialisation betont. Je mehr dagegen Prozesse der Selbstorganisation, Selbstregulierung und Eigenkonstruktivität betont werden, desto mehr kommen Aspekte der Selbstsozialisation in den Blick. Theorien der Selbstsozialisation weisen auf die wachsenden Möglichkeiten und Anforderungen hin, den Aufbau der eigenen Persönlichkeit selbst zu gestalten (vgl. Hurrelmann 2002). Im Rahmen traditionell angelegter Sozialisationsforschungen werden Aspekte der Selbstsozialisation auf der Grundlage einer „konstruktivistischen Sozialisationsforschung“ (Grundmann 1999) untersucht. Dabei kann der allgemeine Begriff der Selbstsozialisation auf die konstruktive Eigentätigkeit von Subjekten abgestellt werden. Diese konstruktive Eigentätigkeit spielt in vielfältigen Kontexten der bisherigen Sozialisationsforschung eine wichtige Rolle (vgl. Dollase 1999: 23ff., Zinnecker 2000).

Darüber hinaus kann man die Theorie vom Bezugsproblem des Verhältnisses von Fremd- und Selbstsozialisation ganz auf Selbstsozialisation umstellen. Mit diesem Schritt markiert der systemtheoretische Begriff der Selbstsozialisation einen Paradigmenwechsel in der Sozialisationsforschung (eingehend hierzu: Sutter 2009), der allerdings bislang noch wenig beachtet wurde und kaum zu bemerkenswerten Auswirkungen in diesem Forschungsfeld geführt hat. Der Paradigmenwechsel steht auf der Grundlage des „operativen Konstruktivismus“(Luhmann 1991: 68), demzufolge sich Systeme durch intern aneinander anschließende Operationen von ihrer Umwelt abgrenzen. Der systemtheoretische Begriff der Selbstsozialisation geht davon aus, dass kommunikative, soziale Systeme und psychische Systeme voneinander getrennt und überschneidungsfrei operieren. Soziale Systeme schließen Kommunikationen an Kommunikationen an, psychische Systeme schließen Gedanken an Gedanken an. Weder können Kommunikationen in Gedanken direkt eingreifen, noch können Gedanken direkt in Kommunikationen eingreifen. Dieser grundlegende Umstand ergibt sich aus der operativen Geschlossenheit sozialer und psy- 
chischer Systeme. Aber diese Geschlossenheit darf nicht verabsolutiert werden, denn es ist diese Verabsolutierung, die traditionell gestimmte Denker seit jeher zu dem Kurzschluss führt, die Systemtheorie habe zur Sozialisation grundsätzlich nichts zu sagen, weil sie „den Menschen“ aus „der Gesellschaft“" und damit aus ihrem Gegenstandsbereich ausschließe. Überschneidungsfreies Operieren sozialer und psychischer Systeme und die Unmöglichkeit, in die jeweils anderen Operationen direkt einzugreifen, bedeuten beileibe keine völlige Isolierung und Abschließung der Systeme, die in vielfältiger und grundlegender Weise auf Umweltkontakte angewiesen sind. Diese Kontakte nehmen nur nicht die Qualität von direkten Einflüssen, Instruktionen, Steuerungen usw. an, sondern stellen Störungen, Irritationen und wechselseitige Leistungsbeziehungen dar, die als unterschiedliche Arten ,struktureller Kopplungen“ von Systemen beschrieben werden können (vgl. Luhmann 1984: 141; Sutter 2009: 93ff.). Es gibt also einen engen Zusammenhang von Geschlossenheit und Offenheit sozialer und psychischer Systeme, der allerdings nicht in ein wechselseitiges Ergänzungsverhältnis von Fremd- und Selbstsozialisation überführt werden kann: Selbstsozialisation, systemtheoretisch gewendet, passt nicht in Vorstellungen einer Vermittlung von Individuum und Gesellschaft. Wenn die Systemtheorie von der operativen Eigenständigkeit subjektiver und sozialer Prozesse ausgeht, und wenn es auf dieser operativen Ebene keine Überschneidung oder Vermittlung, sondern nur Geschlossenheit der Systeme gibt, kann die Sozialisation als Reproduktion psychischer Systeme immer nur Selbstsozialisation sein. Prozesse der Selbstsozialisation sind indessen auf der Ebene der Strukturen in fundamentaler Weise auf Offenheit und Kontakte zu anderen Systemen angewiesen. Grundlegend für jede Sozialisationstheorie ist hier: Ohne Bewusstsein kann sich keine Kommunikation und ohne Kommunikation kein Bewusstsein ausbilden, psychische und soziale Systeme sind konstitutiv voneinander abhängig (vgl. Luhmann 1988).

Aus traditioneller Sicht erscheint die Systemtheorie der Selbstsozialisation als ,Anti-Sozialisationstheorie, die alles abschneidet, was je mit diesem Begriff gemeint war" (Schulze/Künzler 1991: 135). Das vergrößert verständlicherweise die Schwierigkeit, diese Theorie im Feld der Sozialisationsforschung zu etablieren, obwohl sie durchaus gute Gründe ins Feld führen kann. Neben der allgemeinen konstruktivistischen Grundlage bildet das Verhältnis von Gesellschaftsanalyse und Sozialisationstheorie ein weiteres, spezifisch soziologisches Fundament einer Theorie der Selbstsozialisation. Die genannte Umorientierung mag vielen ausgesprochen kontraintuitiv vorkommen, allerdings reagiert die Systemtheorie der Selbstsozialisation auf die zunehmende Komplexität sozialer, ge- 
sellschaftlicher Ausdifferenzierung. Damit wird insgesamt das Verhältnis zwischen Gesellschaftsanalyse und Sozialisationstheorie neu austariert. Die gesellschaftliche Ausdifferenzierung wird vor allem im Rahmen der sozialstrukturellen Sozialisationsforschung in den Blick genommen, die untersucht, wie soziale Strukturen die Persönlichkeitsentwicklung beeinflussen. Die sozialstrukturelle Sozialisationsforschung hat jedoch Mühe, die Beziehungen zwischen gesellschaftlicher Differenzierung und der Persönlichkeitsentwicklung in der modernen Gesellschaft zu erfassen. Auf sozialstruktureller Ebene stellt sich vor allem die Aufgabe, Modelle hierarchischer sozialer Schichtung zu überwinden (vgl. Steinkamp 1991: 275f.). Angesichts der fortschreitenden gesellschaftlichen Ausdifferenzierung erscheint dies dringend geboten: Mit der These zunehmender Individualisierung und der Auflösung industriegesellschaftlicher Lebensformen in der Moderne ist Bewegung in die Sozialstrukturanalyse gekommen. Es genügt nicht mehr, die soziale Lage der Gesellschaftsmitglieder nach Klasse oder Schichtung zu bestimmen, wenn man die Vereinheitlichung der Lebensbedingungen, die Entschichtung der Lebenswelt, horizontale Differenzierungen der Soziallagen und die Pluralisierung von Lebensweisen und Lebensstilen nicht ignorieren will (vgl. Beck 1986, Geißler 1996). Diese schon länger virulenten allgemeinen Probleme der Konzeption sozialer Lagen der Gesellschaftsmitglieder sind im Zusammenhang gesellschaftlicher Differenzierungsprozesse zu sehen. Sie reflektieren insbesondere den Übergang von stratifikatorischer zu funktionaler Differenzierung: Stratifikatorische Differenzierung unterscheidet ungleichrangige und ungleichartige Teile bzw. Teilsysteme (vgl. Luhmann 1997: 613; Schimank 2000: 150ff.). Das bedeutet eine Differenzierung nach oben und unten sowie nach Zentrum und Peripherie. Vor allem die Unterscheidung nach oben und unten ist in der schichtspezifischen Sozialisationsforschung relevant. Nun gibt es nach wie vor arme und reiche, gebildete und weniger gebildete, statushöhere und -niedrigere Personen in der modernen Gesellschaft, also stratifikatorische Differenzierung. Aber diese Differenzierungsform wird in der modernen Gesellschaft durch die mittlerweile dominante Form der funktionalen Differenzierung überlagert.

Die moderne Gesellschaft lässt sich deshalb nicht mehr in Relationen von Teilen und Ganzem oder von Austauschprozessen zwischen Innen und Außen fassen (vgl. Luhmann 1984: 20ff.). Im Zuge der funktionalen Ausdifferenzierung entsteht eine Gesellschaft, die kein einheitliches soziales Gebilde mehr darstellt, in das Menschen einsozialisiert werden könnten. Im Prozess der funktionalen Ausdifferenzierung schließen sich gesellschaftliche Teilsysteme mit je spezifischen Codierungen ab (Wissenschaft nach wahr/unwahr, Recht nach recht/unrecht usw.) und entlas- 
sen die modernen Subjekte in eine azentrische Gesellschaft, die keinen einheitlichen (normativen) Orientierungsrahmen, sondern Möglichkeiten vielfältiger Lebensentwürfe und damit zusammenhängend gesteigerte Chancen und Risiken bietet (vgl. Schimank 2000). Damit steht die Sozialisationstheorie vor der Aufgabe, sich auf Prozesse der funktionalen Differenzierung der modernen Gesellschaft einzustellen (vgl. Sutter 2003). Eine Theorie der Selbstsozialisation kann dabei durchaus auf bewährte Forschungstraditionen etwa in der Tradition Jean Piagets zurückgreifen, dessen Entwicklungstheorie mit der Betonung selbstregulativer Prozesse der Subjektwerdung als Theorie der Selbstsozialisation angesehen werden kann. Das Konzept der Selbstsozialisation könnte nicht nur eine innovative, sondern auch umfassende Perspektive eröffnen, weil sich der Gedanke selbstgesteuerter subjektiver Bildungsprozesse in vielen Entwicklungs- und Sozialisationstheorien findet.

In diesem allgemeinen Rahmen sind Prozesse der Selbstsozialisation im Umgang mit Medien zu analysieren. Diese Prozesse werden aus unterschiedlichen Perspektiven schon seit längerer Zeit eingehend beschrieben und diskutiert (vgl. z.B. Fromme u.a. 1999). In der Regel rechnet man dabei mit einer wichtigen Rolle von Fremdsozialisation:

„Mediennutzung als Selbstsozialisation bedeutet, dass die Sozialisanden die Wahl von Medien und Medieninhalten selbst steuern, über Medienzeiten und Medienorte in relativer Autonomie entscheiden und die Bedeutung der Medieninhalte im Rezeptionsprozess eigenständig konstruieren. Fremdsozialisation bedeutet hier, dass andere Personen oder Institutionen versuchen, den Medienumgang der Heranwachsenden zu lenken im Hinblick auf fremdbestimmte Sozialisationsziele“" (Süss 2004: 67). Aus dieser Sicht gehen Subjekte zwar durchaus autonom und eigenständig mit Medienangeboten um, werden aber auch in vielfältiger Weise beeinflusst, instruiert und gesteuert. Das Verhältnis von Medienangeboten und Rezipienten variiert zwischen den Polen von medialem Zwang und autonomen subjektiven Umgangsweisen mit Medien. Die soziologische Systemtheorie bettet dieses Verhältnis in eine Analyse der funktionalen Ausdifferenzierung des Mediensystems ein, die in die markante Formel mündet: Freiheit auf beiden Seiten (vgl. Luhmann 1996). Die Selbstreferenz und Eigenlogik sowohl medialer Kommunikationsprozesse als auch von Rezeptionsprozessen schließen starke Wirkungen sowie Zwangs- und Manipulationsverhältnisse nicht aus, machen sie aber unwahrscheinlicher und begründungsbedürftiger.

Insofern ist die sozialisationstheoretische Position der Systemtheorie für die Medienpädagogik insgesamt und eine Theorie der Medienbildung besonders bedeutsam, weil sie eine radikal veränderte Sicht auf das Ver- 
hältnis zwischen Medien und Rezipienten begründet. Rezipienten bzw. Mediennutzer werden durch Medien nicht direkt beeinflusst, belehrt, gesteuert, manipuliert, gefördert, gebildet usw., sondern Rezipienten nutzen Medien immer nur nach Maßgabe eigener Möglichkeiten, Fähigkeiten, Themen, Interessen usw. Im Gegenzug orientieren sich die Medien an eigenen Erfordernissen, die auch das Bild der Adressaten medialer Angebote bestimmen. Die Gegenüberstellung von medien- und publikumszentrierten Perspektiven in der Massenkommunikationsforschung, in der immer wieder die Macht der Medien gegen die Autonomie der Rezipienten ausgespielt wurden, wird durch die Systemtheorie der Medien überwunden. Die oben genannte Freiheit auf beiden Seiten ist eng mit Beschränkungen verbunden, die in den beteiligten Systemen etabliert werden. Es herrscht eine Eigenlogik auf beiden Seiten, die strukturell auf Außenweltgegebenheiten ausgerichtet wird. Auf beiden Seiten werden Schemata entwickelt (vgl. ebd.), im Bereich der Massenmedien etwa die bekannten Nachrichtenwerte, an denen sich die Auswahl bzw. Aufbereitung attraktiver Nachrichten ausrichtet. Auch das Publikum wird im Rahmen operativ geschlossener Konstruktionen der Massenmedien beobachtet: Es besteht aus Rezipienten, die nicht direkt erreichbar und beobachtbar sind. Die Massenkommunikation muss sich deshalb selbst ein (kommunikativ konstruiertes!) Bild relevanter Adressatenkreise machen (vgl. Sutter 2010: 70ff.). So setzen die üblicherweise unterschiedenen Programmformen des Fernsehens (Nachrichten, Werbung und Unterhaltung) bestimmte Adressaten voraus: als interessierte Beobachter, als nutzenmaximierende oder sich mit sich selbst auseinandersetzende Personen (vgl. Luhmann 1996: 130ff.). Diese Schematisierungen werden von den Adressaten durch den Umgang mit Medien erworben. Die Massenkommunikation sichert somit gewissermaßen in Eigenregie und mit den vorhandenen Bordmitteln sowohl die Verständlichkeit der Medienangebote als auch die Abnahmebereitschaft der Rezipienten. Dadurch wird nicht determiniert, wie die sich selbst sozialisierenden Rezipienten im Rahmen ihrer persönlichen (kognitiven, affektiven, sprachlichen oder auch moralischen) Voraussetzungen, Erfordernisse und Möglichkeiten mit Medienangeboten umgehen. Die „Wirkung“ eines Medienangebots ist immer nur als Ergebnis aktiver Konstruktions- und Deutungsleistungen der Subjekte zu fassen: Diese allgemeine Einsicht teilen unterschiedliche konstruktivistische Theorien der Mediensozialisation. Die Unterschiede liegen, wie gezeigt, in der Konzeption des Verhältnisses von Fremd- und Selbstsozialisation. 


\section{Selbstsozialisation und neue Medien}

Wie wir gesehen haben kann eine Theorie der Selbstsozialisation durch Medien auf zwei Ebenen angelegt werden: Auf einer allgemeinen systemtheoretischen Ebene kann grundlegend für Selbstsozialisation optiert werden, auf einer gesellschaftsanalytischen Ebene kann die wachsende Bedeutung von Selbstsozialisation im Zuge fortschreitender gesellschaftlicher Differenzierung gezeigt werden. Diese Differenzierung zeigt sich als (medial ermöglichte) Dynamisierung und Beschleunigung gesellschaftlicher Kommunikation (vgl. Münch 1995), die eine Ausdifferenzierung des Mediensystems zur Grundlage hat. In Zeiten beschleunigter medialer Entwicklungen sind Individuen und nachwachsende Generationen zunehmend auf die eigenständige Bewältigung des Umgangs mit Medien angewiesen. Diese beiden Ebenen können vor allem im Rahmen des aktuellen Medienwandels gewinnbringend miteinander verbunden werden: die allgemeine und grundlegend gegebene Bedeutung von Selbstsozialisation im Umgang mit Medien könnte im Bereich neuer Medien deutlich an Gewicht zunehmen. Die Frage ist, ob ein Zusammenhang zwischen dem aktuellen Medienwandel und einer gesteigerten Bedeutung von Selbstsozialisation plausibel gemacht werden kann. In dieser Frage wird man zunächst einmal Vorsicht walten lassen. Während die älteren Massenmedien seit je eher für Prozesse und Auswirkungen von Fremdsozialisation in Form unterschiedlicher Zwänge stehen, werden neue Medien - oft ebenso pauschal - als neue, individuell nutzbare Freiräume gesehen.

Der Grund liegt in einem deutlich positiv gefärbten Bild, das man von dem aktuellen, tiefgreifenden Wandel von älteren Massenmedien hin zu neuen ,interaktiven“ Kommunikationsmedien zeichnet. Im Hinblick auf die vielfach geäußerten Kritiken vor allem am Leitmedium Fernsehen, das die Zuschauer in einer passiven Rolle auf Distanz hält, aus der heraus weder reagiert noch eingegriffen oder mitgestaltet werden kann, wird der Medienwandel meist positiv gedeutet: neue, computergestützte Kommunikationsmedien befreien uns von den Zwängen der Massenmedien durch vielfältige Rückkopplungs- und Mitwirkungsmöglichkeiten. Zwar lauern auch hier Risiken und Gefahren, und nach den PC-Spielen sind nun auch Netzwerkplattformen wie Facebook in der Diskussion. Aber die hoffnungsfrohen, wenn nicht gar illusionären Erwartungen an das Internet übersteigen deutlich die Kritiken. Im vorliegenden Zusammenhang ist zu fragen, was eigentlich das Neue der ,,neuen Medien“ sein soll und welche Auswirkungen neue Medien auf Prozesse der Sozialisation haben.

In Abhebung von älteren Massenmedien eröffnen neue ,interaktive“ Medien vielfältige Rückkopplungs- und Eingriffsmöglichkeiten für die 
Nutzer, was vor allem unter Stichworten wie „Web 2.0“ und „Social Web“ beschrieben wird. Wie immer man diese Entwicklung einschätzt, sicherlich eröffnen viele Internetanwendungen einen individuell gestaltbaren Zugang zu Medienangeboten. Deshalb kann man fragen, wie diese neuen individuellen Umgangsweisen mit Medienangeboten gelernt werden, ob also die gewandelten Formen der Medien Auswirkungen auf Prozesse der Mediensozialisation haben. Aus Sicht der bisherigen Überlegungen kann die zentrale These hierzu nur lauten: Wenn und soweit hier Auswirkungen zu beobachten sind, müssten die als eine gesteigerte Bedeutung von Selbstsozialisation in den Blick kommen. Wenn im Zuge der Enttraditionalisierung, Pluralisierung, Individualisierung und Vielfalt von Identitäts- und Lebensentwürfen Prozesse der Sozialisation zunehmend in Eigenregie der Individuen verlaufen, so erscheinen die neuen Möglichkeiten vieler Internetanwendungen als neue, machtvolle Taktgeber dieser Dynamik. Gerade die neuen Medien kommen diesen Tendenzen entgegen, indem sie die Eingriffs- und Gestaltungsmöglichkeiten für die Nutzer und damit die Autonomie im Umgang mit Medien steigern. Das Internet bietet neue Möglichkeiten, Identitäts- und Lebensentwürfe spielerisch auszuprobieren: Stichworte hierzu sind Maskierung, Anonymität, neue (unverbindliche) Kommunikationsmöglichkeiten, weitgespannte Vernetzung, vielfältige neue Kontakte, neue Formen der Gemeinschaft usw. Wie schon die älteren Massenmedien bieten auch die neuen Medien Chancen und Risiken: Man kann sich in den spielerischen, unverbindlichen Entwürfen verlieren oder aber sie für eine gesteigerte Reflexion nutzen. Das gilt vor allem für die sozialen Netzwerke, die neue, faszinierende Möglichkeiten der Identitätsdarstellung und -präsentation eröffnen. Neu sind hierbei allerdings auch die erheblichen Risiken der Veröffentlichung von Privatem, die erst nach und nach ins allgemeine Bewusstsein der Nutzer gehoben und ausgelotet werden.

Einmal mehr erweist sich damit ein Komplex sozialwissenschaftlicher Medienforschungen insgesamt und auch speziell der Mediensozialisationsforschung als zentrale Grundlage: die Kontexte der Mediennutzung. Es kommt nicht nur darauf an, die Bedeutung bzw. Eigenlogik von Medien(angeboten) an sich zu analysieren, sondern auch deren Umsetzung und Realisation. Die Möglichkeiten und Risiken unterschiedlicher Internetanwendungen werden ganz unterschiedlich realisiert. Wenn das Internet prinzipiell weit mehr Beteiligungsmöglichkeiten für die Nutzer eröffnet als die älteren Massenmedien, so bedeutet das noch nicht, dass diese Möglichkeiten auch wahrgenommen werden. Allgemein wird beobachtet, dass das Interesse an aktiver Teilhabe im Rahmen von Web 2.0-Anwendungen auf niedrigem Niveau stagniert (vgl. Busemann/Gscheidle 
2011). Eine wichtige Ausnahme hiervon sind die privat genutzten sozialen Netzwerke. Hier werden viele Möglichkeiten der Vernetzung und Kommunikation genutzt, aber auf sehr unterschiedliche Weise. Viele Kontakte und die geforderte ständige Erreichbarkeit können zu oberflächlichen, wenig bedeutsamen Beziehungen („Freunden“) führen. Der entscheidende Unterschied ist, ob man sich in diese Beziehungen flüchtet oder bestehende Beziehungen per Internet erweitert. $\mathrm{Zu}$ beobachten sind vor allem Auswirkungen der Nutzung von Social Media auf die Sozialisation in Gleichaltrigengruppen, was als ,digitale Jugendkulturen“ beschrieben wird (vgl. Hugger 2010). Jugendliche betreten neue Wege der Selbstdarstellung, Selbstverortung, Selbstinszenierung, und Selbstreflexion, kurz: der „Identitätsarbeit“. Auf diesen Wegen trifft man auf neue Probleme, deren Bedeutung und Lösung noch nicht abzusehen sind. $\mathrm{Zu}$ nennen sind hier vor allem private Daten, die ins Netz eingegeben werden, und die gespeichert, weiter verbreitet und jederzeit durchsucht werden können. Insgesamt stellen sich in diesen neuen medialen Kommunikationsräumen für die Nachwachsenden Probleme gesteigerter Unsicherheiten und Intransparenzen. Bei der Bewältigung dieser Probleme durchlaufen Jugendliche zunehmend Prozesse der Selbstsozialisation, wobei vormals festgefügte Vorgaben zur Disposition stehen. Damit wächst die Bedeutung des selbstgesteuerten Umgangs der Nachwachsenden mit neuen Medien.

\section{Selbstsozialisation und Medienbildung}

Der soziologische Beitrag zu einer Theorie der Medienbildung kann an dieser Stelle auf den genannten Linien nur programmatisch umrissen werden und setzt einen anderen Akzent als die medienpädagogische Debatte. Statt relativ voraussetzungsreich und konkret ansetzende kompetenztheoretische, bildungspolitische oder kulturkritische Konzeptionen ins Feld zu führen ${ }^{1}$, tritt man gleich mehrere Schritte zurück, um allgemeine sozialisations- und gesellschaftstheoretische Grundlagen in den Blick zu bekommen. Zu berücksichtigen sind dabei die selbstreferentiellen, eigenlogischen Dynamiken und Strukturen der gesellschaftlichen Ausdifferenzierung, damit verbunden des beschleunigten Medienwandels und der Prozesse der Selbstsozialisation. Eine deutliche Zuspitzung erfährt dieser Zusammenhang mit der These einer gesteigerten Bedeutung von Prozessen der Selbstsozialisation im Umgang mit neuen, computergestützten

\footnotetext{
1 Dies betrifft allerdings nicht weit ausgreifende theoretische Zugänge zur Medienbildung, wonach im Kontext eines weiten Medienbegriffs sich Bildung grundsätzlich als Medienbildung vollzieht: in diesem Sinne Meder (2011), in einer ähnlichen Richtung Spanhel (2010).
} 
Medien. Damit sind steuerungs-, instruktions- und planungsbezogene Konzepte von Medienbildung, die auf Lernzielkataloge und bildungspolitische Strategien ausgerichtet sind, wenig aussichtsreich. Es muss dann auch neu gefragt werden, wo und wie Prozesse der Medienbildung überhaupt gefördert werden können. Jenseits denkbarer Bildungsplanungen vollzieht sich das ohnehin vor allem in selbst organisierten Gruppen von Gleichaltrigen bzw. Nutzungsgemeinschaften im Internet. Eltern und Schule dagegen scheitern oft schon an der schieren Geschwindigkeit der Medienentwicklung, so dass Kinder und Jugendliche vielfach sich selbst überlassen sind. Außerdem laden viele Internetanwendungen zum Erkunden und Ausprobieren jenseits kontrollierbarer Umgangsweisen mit vorgegebenen Inhalten ein. Web 2.0 bedeutet gerade keine Bereitstellung identischer Lernmaterialien, die auf nachvollziehbare und nachprüfbare Weise angeeignet werden. $\mathrm{Ob}$ sich die Schule auf das Wagnis Internet einlassen kann, ist nicht nur eine Frage der Bereitschaft und Fähigkeit von Lehrern und der Ausstattung von Schulen.

Wenn die These einer ohnehin grundlegend gegebenen und im Umgang mit neuen Medien gesteigerten Bedeutung von Selbstsozialisation plausibel ist, wird man Konzeptionen von Medienbildung darauf abstellen müssen. Im Zusammenhang damit sind immer wieder Vorurteile und ideologische Scheuklappen in der Frage zu beseitigen, ob und in welcher Weise die Mediennutzung zur Bildung beiträgt. Auf der Linie der vorlaufenden Erörterungen könnte eine Theorie der Medienbildung als eine Art Reflexionstheorie fungieren, die unter den genannten Bezugsproblemen der Bedeutung von Selbstsozialisation in der modernen, funktional ausdifferenzierten Gesellschaft die Möglichkeiten und Leistungen von Medien und des Umgangs mit Medien für subjektive Bildungsprozesse untersucht. Schon bei der allgemeinen Einschätzung von Beobachtungs- und Reflexionsmöglichkeiten gehen die Auffassungen auseinander. Wenn man etwa dem Massenmedium Fernsehen aufgrund seiner einseitigen Form vielfältige direkte (und zumeist negativ konnotierte) Einflussmöglichkeiten zuschreibt, verkennt man die spezifischen Reflexionsmöglichkeiten, die gerade die handlungsentlasteten, distanzierten Beobachtungsmöglichkeiten von Fernsehsendungen bieten (vgl. Wehner 1997). In diesem Sinne eröffnet das Fernsehen unverzichtbare Gelegenheiten der Reflexion von eigenen Wissensbeständen sowie Lebens- und Identitätsentwürfen. Dies zeigen auch rekonstruktive Rezeptions- und Sozialisationsforschungen (vgl. Charlton 1997), und davon bleiben viele der behaupteten negativen Wirkungen des Fernsehkonsums unbenommen. Dagegen können Internetanwendungen gerade aufgrund ihrer „Interaktivität“ (vgl. Sutter 2010: 154ff.) diese Reflexionsmöglichkeiten des unbeteiligten, distanzierten 
Beobachtens einschränken. Das kritisch zu betrachtende, weil einseitig kommunizierende und dadurch passive Rezipienten beeinflussende Fernsehen hier, das hoffnungsfroh stimmende, weil zur aktiven Beteiligung ermunternde Internet dort: so einfach liegen die Dinge nicht. Wenn man einmal die Probleme und Sichtweisen in Bewegung gebracht hat, stößt jede Behauptung auf Skepsis und Alternativen.

Eine Reflexionstheorie der Medienbildung wird man deshalb mit guten Gründen nicht auf materiale, sondern prozessuale Gesichtspunkte ausrichten, wie das explizit auch die Position der ,Strukturalen Medienbildung“ hervorhebt (vgl. Marotzki/Jörissen 2010). Eine Veränderung von Selbst- und Weltverhältnissen, eine Steigerung von Reflexivität und der Aufbau von „Orientierungswissen“ (ebd.) sind in Beziehung zu den Strukturen der Medien zu untersuchen. Das gilt insbesondere im Bereich neuer, digitaler Medien (vgl. Zorn 2011), die Ergebnis eines tiefgreifenden Medienwandels sind, der neue mediale Formen und neue subjektive Umgangsweisen mit Medien hervorbringt. Allgemein geht es um Kopplungsbeziehungen zwischen den Sinn- und Bedeutungsstrukturen der Medien, den vielfältigen sozialen Kontexten der Mediennutzung und den subjektiven Umgangsweisen mit Medien. Ein zunehmend ausdifferenziertes System von Massenmedien und Internetanwendungen stellt ganz neue Herausforderungen, die im Zuge der Mediensozialisation bewältigt werden müssen. Wenn man beispielsweise um die Gefahren der Speicherung, Veröffentlichung und Verbreitung persönlicher Daten in Facebook weiß, so ist damit die Bedeutung dieses Wissens in der konkreten persönlichen Lebenssituation nicht festgelegt: Man könnte es sich etwa in der Gleichaltrigengruppe nur schwer leisten, auf die Teilnahme an Facebook zu verzichten, und sieht sich gezwungen, die Risiken in Kauf zu nehmen.

Der Umgang mit Medien und damit zusammenhängende Fragen der Medienbildung sind methodologisch auf eingehende, strukturrekonstruktiv vorgehende Einzelfallanalysen angewiesen. Ebenso wenig wie mit den lange Zeit dominierenden Inhaltsanalysen im Bereich der Massenkommunikationsforschung wird man auf Dauer mit den derzeit verbreiteten ethnographischen Vorgehensweisen im Bereich neuer Medien auskommen. Zwar ist es wichtig und richtig, erst einmal zu beschreiben, was in den neuen medialen Kommunikationsräumen vor sich geht, aber das ersetzt keine kontextsensitiven Explikationen von Bedeutungen. Wir haben schon eine recht gute Vorstellung davon, welche strukturellen Merkmale die Eigenlogik neuer Medien bestimmen, so etwa die Anonymität der Adressaten, gesteigerte Kontingenzen durch die Zwischenschaltung informationsverarbeitender Maschinen, neue Formen von Beziehungen zwischen Personen und Texten sowie zwischen Texten, das Auseinan- 
derziehen von Mitteilungs- und Verstehensprozessen und eine damit verbundene Individualisierung der Sequentialität des Geschehens (vgl. Sutter 2010: 160ff.). Die Frage, welche Bedeutung diese Merkmale für die Sozialisation und Bildung von Subjekten haben, wird in strukturrekonstruktiven Kommunikationsanalysen zu bearbeiten sein. Dass hieraus nur indirekt auf subjektive Bildungsprozesse geschlossen werden kann, ist ein allgemeines methodologisches Problem, das allerdings aus der hier eingenommenen konstruktivistischen Position besonders deutlich gemacht werden kann. Mit Verfahren wie der objektiven Hermeneutik oder der Konversationsanalyse hat man etablierte Methoden zur Hand, die erfolgreich mit den Vorzügen und Problemen eines strikt kommunikationsanalytischen Vorgehens umgehen. Vor eine weitere Schwierigkeit sind Reflexionstheorien und (zeitaufwändige) rekonstruktive Methoden speziell im Bereich der Mediensozialisation und Medienbildung gestellt: mit dem rasanten Medienwandel Schritt zu halten. Einen zentralen Vorzug einer Theorie der Medienbildung würde ich darin sehen, genau das erst gar nicht zu versuchen und umso sorgfältiger die relevanten Fragen und Bezugsprobleme zu erarbeiten.

\section{Literatur:}

Beck, Ulrich (1986): Risikogesellschaft. Auf dem Weg in eine andere Moderne. Frankfurt a. M. (Suhrkamp).

Busemann, Katrin/Gscheidle, Christoph (2011): Web 2.0: Aktive Mitwirkung verbleibt auf niedrigem Niveau. Ergebnisse der ARD/ZDF-Online-Studie 2011. In: Media Perspektiven, Jg. 15, H. 7/8. S. 360-369.

Charlton, Michael (1997): Rezeptionsforschung als Aufgabe einer interdisziplinären Medienwissenschaft. In: Charlton, Michael/Schneider, Silvia (Hrsg.): Rezeptionsforschung. Theorien und Untersuchungen zum Umgang mit Massenmedien. Opladen (Westdeutscher Verlag). S. 16-39.

Chomsky, Noam (1972): Aspekte der Syntax-Theorie. Frankfurt a. M. (Suhrkamp).

Dollase, Rainer (1999): Selbstsozialisation und problematische Folgen. In: Fromme, Johannes/Kommer, Sven/Mansel, Jürgen/Treumann, Klaus Peter (Hrsg.): Selbstsozialisation, Kinderkultur und Mediennutzung. Opladen (Leske + Budrich). S. 23-42.

Fromme, Johannes/Kommer, Sven/Mansel, Jürgen/Treumann, Klaus Peter (Hrsg.) (1999): Selbstsozisalisation, Kinderkultur und Mediennutzung. Opladen (Leske + Budrich) 
Geißler, Rainer (1996): Die Sozialstruktur Deutschlands. Zur gesellschaftlichen Entwicklung mit einer Zwischenbilanz zur Vereinigung. Opladen (Westdeutscher Verlag).

Grundmann, Matthias (Hrsg.) (1999): Konstruktivistische Sozialisationsforschung: Ansätze und methodische Überlegungen zu einer konstruktivistischen Theorie der Sozialisation. Frankfurt a.M. (Suhrkamp).

Habermas, Jürgen (1995): Notizen zur Entwicklung der Interaktionskompetenz. In: Habermas, Jürgen.: Vorstudien und Ergänzungen zur Theorie des kommunikativen Handelns. Frankfurt a. M. (Suhrkamp). S. 187-225.

Herzig, Bardo/Grafe, Silke (2010): Entwicklung von Bildungsstandards für die Medienbildung - Grundlagen und Beispiele. In: Herzig, Bardo/ Meister, Dorothee M./Moser, Heinz/Niesyto Horst (Hrsg.): Jahrbuch Medienpädagogik 8: Medienkompetenz und Web 2.0. Wiesbaden: (VS Verlag für Sozialwissenschaften). S. 41-58.

Hugger, Kai-Uwe (2010): Digitale Jugendkulturen. Wiesbaden (VS Verlag für Sozialwissenschaften).

Hurrelmann, Klaus (2002): Selbstsozialisation oder Selbstorganisation? Ein sympathisierender, aber kritischer Kommentar. In: Zeitschrift für Soziologie der Erziehung und Sozialisation, Jg. 22, H. 2. S. 155-166. Jörissen, Benjamin (2011): „Medienbildung“. Begriffsverständnisse und -reichweiten. In: Grell, Petra/Moser, Heinz/Niesyto, Horst (Hrsg.): Medienbildung und Medienkompetenz. Beiträge zu Schlüsselbegriffen der Medienpädagogik. München (kopaed). S. 211-235.

Luhmann, Niklas (1984): Soziale Systeme. Grundriß einer allgemeinen Theorie. Frankfurt a. M. (Suhrkamp).

Luhmann, Niklas (1988): Wie ist Bewußtsein an Kommunikation beteiligt? In: Gumbrecht, Hans Ulrich/Pfeiffer, K. Ludwig (Hrsg.): Materialität der Kommunikation. Frankfurt a. M. (Suhrkamp). S. 884-905. Luhmann, Niklas (1991): Wie lassen sich latente Strukturen beobachten? In: Krieg, Peter/Watzlawick, Paul (Hrsg.): Das Auge des Betrachters. Beiträge zum Konstruktivismus. München, Zürich (Piper) S. 61-74.

Luhmann, Niklas (1996): Die Realität der Massenmedien. 2. erweiterte Auflage. Opladen (Westdeutscher Verlag).

Luhmann, Niklas (1997): Die Gesellschaft der Gesellschaft, 2 Bände. Frankfurt a. M. (Suhrkamp).

Marotzki, Winfried/Jörissen, Benjamin (2010): Dimensionen strukturaler Medienbildung. In: Herzig, Bardo/Meister, Dorothee M./Moser, Heinz/Niesyto, Horst (Hrsg.): Jahrbuch Medienpädagogik 8: Medienkompetenz und Web 2.0. Wiesbaden (VS Verlag für Sozialwissenschaften). S. 19-39. 
Meder, Norbert (2011): Von der Theorie der Medienpädagogik zu einer Theorie der Medienbildung. In: Fromme, Johannes/Iske, Stefan/Marotzki, Winfried (Hrsg.): Medialität und Realität. Zur konstitutiven Kraft der Medien. Wiesbaden (VS Verlag für Sozialwissenschaften). S. 67-81.

Münch, Richard (1995): Dynamik der Kommunikationsgesellschaft. Frankfurt a. M. (Suhrkamp).

Pietraß, Manuela (2009): Medienbildung. In: Schmidt, Bernd/Tippelt, Rudolf (Hrsg.): Handbuch Bildungsforschung. 3. überarbeitete Ausgabe. Wiesbaden (VS Verlag für Sozialwissenschaften). S. 499-512.

Schimank, Uwe (2000): Theorien gesellschaftlicher Differenzierung. 2. Auflage. Opladen (Leske + Budrich).

Schulze, Hans-Joachim/Künzler, Jan (1991): Funktionalistische und systemtheoretische Ansätze in der Sozialisationsforschung. In: Hurrelmann, Klaus/Ulich, Dieter (Hrsg.): Neues Handbuch der Sozialisationsforschung. Weinheim, Basel (Beltz). S. 121-136.

Spanhel, Dieter (2010): Bildung in der Mediengesellschaft. Medienbildung als Grundbegriff der Medienpädagogik. In: Bachmair, Ben (Hrsg.): Medienbildung in neuen Kulturräumen. Die deutschsprachige und britische Diskussion. Wiesbaden (VS Verlag für Sozialwissenschaften). S. 45-58.

Steinkamp, Günther (1991): Sozialstruktur und Sozialisation. In: Hurrelmann, Klaus/Ulich, Dieter (Hrsg.): Neues Handbuch der Sozialisationsforschung. Weinheim, Basel (Beltz). S. 251-277.

Süss, Daniel (2004): Mediensozialisation von Heranwachsenden: Dimensionen - Konstanten - Wandel. Wiesbaden (VS Verlag für Sozialwissenschaften).

Sutter, Tilmann (1999): Bausteine einer konstruktivistischen Theorie der Mediensozialisation. In: Fromme, Johannes/Kommer, Sven/Mansel, Jürgen/Treumann, Klaus-Peter (Hrsg.): Selbstsozialisation, Kinderkultur und Mediennutzung. Opladen (Leske + Budrich). S. 126-138.

Sutter, Tilmann (2003): Sozialisationstheorie und Gesellschaftsanalyse. Zur Wiederbelebung eines zentralen soziologischen Forschungsfeldes. In: Bretzinger, Bettina/Holz, Klaus/Wenzel, Ulrich (Hrsg.): Subjekte und Gesellschaft. Zur Konstitution von Sozialität. Weilerswist (Velbrück Wissenschaft). S. 45-69.

Sutter, Tilmann (2009): Interaktionistischer Konstruktivismus. Zur Systemtheorie der Sozialisation. Wiesbaden (VS Verlag für Sozialwissenschaften). 
Sutter, Tilmann (2010): Medienanalyse und Medienkritik. Forschungsfelder einer konstruktivistischen Soziologie der Medien. Wiesbaden (VS Verlag für Sozialwissenschaften).

Sutter, Tilmann/Charlton, Michael (2002): Medienkompetenz - einige Anmerkungen zum Kompetenzbegriff. In: Groeben, Norbert/Hurrelmann Bettina (Hrsg.): Medienkompetenz. Voraussetzungen, Dimensionen, Funktionen. Weinheim (Juventa). S. 129-147.

Theunert, Helga (1999): Medienkompetenz. Eine pädagogisch und altersspezifisch zu fassende Handlungsdimension. In: Schell, Fred/Stolzenburg, Elke/Theunert, Helga (Hrsg.): Medienkompetenz: Grundlagen und pädagogisches Handeln. München (kopaed). S. 50-59.

Wehner, Josef (1997): Interaktive Medien - Ende der Massenkommunikation? In: Zeitschrift für Soziologie, Jg. 26, H. 2. S. 96-114.

Zinnecker, Jürgen (2000): Selbstsozialisation - Essay über ein aktuelles Konzept. Zeitschrift für Sozialisationsforschung und Erziehungssoziologie, Jg. 20, H. 3. S. 272-290.

Zorn, Isabel (2011): Zur konstitutiven Kraft Digitaler Medien: Bildungsrelevanz von Konstruktionstätigkeiten mit Digitalen Medien. In: Fromme, Johannes/Iske, Stefan/Marotzki, Winfried (Hrsg.): Medialität und Realität. Zur konstitutiven Kraft der Medien. Wiesbaden (VS Verlag für Sozialwissenschaften). S. 177-192. 